\title{
Dynamic Interactions of Two Electrical Double Layers
}

\author{
Jyh-Ping Hsu, ${ }^{* 1}$ Yung-Chih Kuo, $*$ and Shiojenn Tseng $\dagger$ \\ * Department of Chemical Engineering, National Taiwan University, Taipei 10617, Taiwan, Republic of China; \\ and $\dagger$ Department of Mathematics, Tamkang University, Tamsui 25137, Taipei, Republic of China
}

Received June 3, 1997; accepted August 28, 1997

The unsteady-state electrical potential and the concentrations of ions between two identical, negatively-charged particles immersed in an a:b electrolyte solution are investigated. In particular, the effects of ionic strength, $I$, the geometric mean of the diffusivities of counterions $D_{\text {con }}$ and coions $D_{\text {co, }} D$, the separation distance between two particles, $H$, and the surface charge density, $\sigma_{0}$, on these distributions are examined. We conclude that under the following conditions a system needs a longer time for ions to reach equilibrium distributions: (a) small $I$, (b) small $D$, (c) large $\boldsymbol{H}$, and (d) large $\sigma_{0}$. The rate of approach of two particles is faster if both surfaces are maintained at constant potential than if both surfaces are at constant charge density. The dynamic behavior of the relaxation of ions in the double layers has the effect of retarding the motion of particles. The deviation in the contact time between two particles predicted by an equilibrium model, which assumes that the distributions of ions in a double layer reach the B oltzmann distribution instantly, from that estimated by the corresponding dynamic model is on the order of $10 \%$. 1997 Academic Press

Key Words: electrical double layer; relaxation; charged surfaces; constant potential; constant charge density; Poisson equation; nonlinear; transient; numerical solution; Nernst- Planck equation.

\section{INTRODUCTION}

There has been a significant progress in the description of the interactions between charged colloidal particles in the last two decades. In particular, the boundary conditions often assumed in the resolution of Poisson-Boltzmann equation (PBE) governing the potential distribution of a colloidal system, i.e., constant surface potential or constant surface charge density, are modified to reflect, more realistically, the physical situation encountered in practice. Ninham and Parsegian (1), for example, considered a charge-regulated surface to simulate biological cells. This model was extended to estimate the effect of multivalent cations on the rate of adhesion of cells to surfaces (2). Attempts have also been made to derive the surface charge density-surface potential relation for spherical and cylindrical surfaces. The improve-

${ }^{1}$ To whom correspondence should be addressed. ment in the computing facilities makes numerical solution of a complicated problem feasible, and approximate analytical solutions to PBE becomes convenient in the estimation of the thermodynamic properties of an electrical double layer $(3,4)$. Previous efforts on the relevant problems, however, almost always assume that the electrical condition near a charged surface is at equilibrium. In particular, the space charges are assumed to establish Boltzmann distribution instantaneously, regardless of the variable condition at the surface $(5,6)$. In a study of the stability of a colloidal system, Overbeek (7) pointed out that the relaxation time for surface charges (about $10^{-6}$ to $10^{4} \mathrm{~s}$ ) can be comparable to the time scale for Brownian coagulation (about $10^{-7}$ to $10^{-5} \mathrm{~s}$ ). In other words, aggregation of colloidal particles may occur before the electrical condition near a surface reaches equilibrium. Kijlstra and van Leeuwen (5) concluded that the temporal variation in the surface charge density has a significant effect on the stability ratio of a colloidal system. In these cases, the steady-state analysis on the electrical condition near a charged surface becomes unrealistic, and an extension of the conventional treatment to a temporal description seems to be inevitable. In a study of the stability of hydrophobic colloids, the dynamic behavior of ions in two interacting electric double layers was discussed by Lyklema (8). It was shown that the rate of approach of particles is reduced significantly due to the dynamic behavior of the double layer (9). The reduction in this rate is comparable to that induced by purely hydrodynamic resistance (10).

The effect of the rate of dissociation of the ionogenic groups on a cell surface on the rate of its adhesion to a solid surface of constant potential was examined recently (6). The rate for space charges to reach equilibrium (Boltzmann) distribution was assumed to be much faster than that of the dissociation of ionogenic groups. It was found that if the ionic strength is on the order of $1 M$, the time scale for the rate of adhesion is about $10^{-8} \mathrm{~s}$. The temporal behavior in the dissociation of ionogenic groups on a cell surface also has a significant effect on the rate of cell adhesion. Assuming that the adsorption-desorption of charge-determining ions is the rate-determining process, Shulepov et al. (11) analyzed the interaction of colloidal particles which takes the 
transient behavior of double layer into account. The desorption rate constant was found to be the most critical characteristic. On the basis of the above discussion, it is apparent that a detailed understanding about the temporal variation in the space charges and its effect on the behavior of a chargeregulated surface deserve further study in the next stage. Here, the behavior of the temporal variations in both electrical potential and space charge distributions are examined. In particular, the time scale to reach a new equilibrium distribution is estimated, and the key factors affecting this scale are discussed. Also, the influence of double-layer relaxation on the relative motion of two colloidal particles is assessed.

\section{ANALYSIS}

The system under consideration comprises two charged surfaces immersed in an a:b electrolyte solution separated by a distance $H$. One of the surfaces is located at the origin. Both surfaces remain at a constant charge density. The distribution of the concentration of species $i$ between surfaces, $C_{i}$, is described by

$$
\frac{\partial C_{i}}{\partial t}=-\nabla \cdot N_{i}
$$

where $t$ is time, $N_{i}$ the mass flux of ion species $i$, and $\nabla$ the gradient operator. Under the conditions normally encountered in practice, $N_{i}$ can be described by the Nernst-Planck equation,

$$
N_{i}=-D_{i} \nabla C_{i}-\left(\frac{Z_{i} F}{R T}\right) D_{i} C_{i} \nabla \phi,
$$

where $D_{i}$ and $Z_{i}$ are, respectively, the diffusivity and the valence of ion species $i, R$ and $F$ are, respectively, the gas constant and the Faraday constant, $T$ is absolute temperature, and $\phi$ denotes the electrical potential. Suppose that two surfaces are sufficiently close to each other, and the system under consideration can be approximated by a one-dimensional problem. Substituting Eq. [2] into Eq. [1] yields

$$
\frac{\partial C_{i}}{\partial t}-D_{i} \frac{\partial^{2} C_{i}}{\partial r^{2}}-D_{i} \frac{Z_{i} F}{R T}\left(C_{i} \frac{\partial^{2} \phi}{\partial r^{2}}+\frac{\partial C_{i}}{\partial r} \frac{\partial \phi}{\partial r}\right)=0,
$$

where $r$ denotes the position variable. For simplicity, we assume that diffusivity is not a function of position. For the present system, the Poisson equation becomes

$$
\frac{\partial^{2} \phi}{\partial r^{2}}=-\frac{\rho_{\mathrm{c}}}{\epsilon_{0} \epsilon_{\mathrm{r}}}
$$

with $\rho_{\mathrm{c}}=\Sigma Z_{i} F C_{i}$. Here, $\rho_{\mathrm{c}}$ represents the space charge density, $\epsilon_{0}$ and $\epsilon_{\mathrm{r}}$ are, respectively, the permittivity of the vacuum and the relative permittivity. For an a:b electrolyte solution, Eqs. [3] and [4] become, respectively,

$$
\begin{gathered}
\frac{\partial \rho_{\mathrm{con}}}{\partial t}-D_{\text {con }} \frac{\partial^{2} \rho_{\text {con }}}{\partial r^{2}}-D_{\text {con }} \frac{a F}{R T}\left(\rho_{\text {con }} \frac{\partial^{2} \phi}{\partial r^{2}}+\frac{\partial \rho_{\mathrm{con}}}{\partial r} \frac{\partial \phi}{\partial r}\right)=0 \\
\frac{\partial \rho_{\mathrm{co}}}{\partial t}-D_{\mathrm{co}} \frac{\partial^{2} \rho_{\mathrm{co}}}{\partial r^{2}}+D_{\mathrm{co}} \frac{b F}{R T}\left(\rho_{\mathrm{co}} \frac{\partial^{2} \phi}{\partial r^{2}}+\frac{\partial \rho_{\mathrm{co}}}{\partial r} \frac{\partial \phi}{\partial r}\right)=0
\end{gathered}
$$

$$
\frac{\partial^{2} \phi}{\partial r^{2}}=-\frac{\rho_{\mathrm{con}}-\rho_{\mathrm{co}}}{\epsilon_{0} \epsilon_{\mathrm{r}}}
$$

In these expressions, $\rho_{\text {con }}$ and $\rho_{\text {co }}$ are, respectively, the charge densities of counterion and coion, $\rho_{\text {con }}=a F C_{\text {con }}$ and $\rho_{\text {co }}=$ $b F C_{\mathrm{co}}, C_{\mathrm{con}}$ and $C_{\mathrm{co}}$ being the concentrations of counterion and coion, respectively, and $D_{\text {con }}$ and $D_{\text {co }}$ are, respectively, the diffusivities of counterion and coion.

For an easier manipulation, a similarity transformation is conducted on Eqs. [5] - [7] through defining a new independent variable $\eta$ as (12)

$$
\eta=\frac{r}{(D t)^{1 / 2}}
$$

After the transformation, Eqs. [5]-[7] reduce to, respectively,

$$
\begin{gathered}
\frac{\eta}{2} \frac{d \xi_{\mathrm{con}}}{d \eta}+\frac{D_{\mathrm{con}}}{D} \frac{d^{2} \xi_{\mathrm{con}}}{d \eta^{2}}+\frac{a D_{\mathrm{con}}}{D}\left(\xi_{\mathrm{con}} \frac{d^{2} \psi}{d \eta^{2}}+\frac{d \xi_{\mathrm{con}}}{d \eta} \frac{d \psi}{d \eta}\right)=0 \\
\frac{\eta}{2} \frac{d \xi_{\mathrm{co}}}{d \eta}+\frac{D_{\mathrm{co}}}{D} \frac{d^{2} \xi_{\mathrm{co}}}{d \eta^{2}}-\frac{b D_{\mathrm{co}}}{D}\left(\xi_{\mathrm{co}} \frac{d^{2} \psi}{d \eta^{2}}+\frac{d \xi_{\mathrm{co}}}{d \eta} \frac{d \psi}{d \eta}\right)=0
\end{gathered}
$$

and

$$
\frac{d^{2} \psi}{d \eta^{2}}=\frac{-\tau}{a+b}\left(\xi_{\mathrm{con}}-\xi_{\mathrm{co}}\right)
$$

In these expressions, $\xi_{\text {con }}=\rho_{\text {con }} / \rho_{0}, \xi_{\text {co }}=\rho_{\text {co }} / \rho_{0}, D=\left(D_{\text {con }}\right.$ $\left.D_{\text {co }}\right)^{1 / 2}, \psi=F \phi / R T, \tau=D t \kappa^{2}, \kappa^{2}=2 I F^{2} / \epsilon_{0} \epsilon_{\mathrm{r}} R T$, and $\rho_{0}$ $=2 I F /(a+b)$. Here $D$ denotes the geometric mean of $D_{\text {con }}$ and $D_{\text {co }}, \tau$ is the scaled time, $\kappa$ is the reciprocal Debye length, $I$ represents the ionic strength of the bulk solution $(\phi \rightarrow 0$ or $H \rightarrow \infty), \rho_{0}$ is the counterion (or coion) charge density in the bulk phase (or as $H \rightarrow \infty$ ), $\xi_{\text {con }}$ and $\xi_{\text {co }}$ are, respectively, the scaled charge densities of counterion and coion, and $\psi$ is the scaled electrical potential. For conve- 
nience, we assume that the ions are at the equilibrium state initially. The subsequent adsorption/desorption and/or the exchange of the potential-determining ions on the surfaces causes the variation in the charged conditions on particle surfaces.

If the surfaces are ion-impenetrable, Eq. [2] leads to

$$
C_{i}=C_{i 0} \exp \left(\frac{-Z_{i} F \phi_{\mathrm{B}}}{R T}\right) \text {, }
$$

where $C_{i 0}$ and $\phi_{\mathrm{B}}$ are, respectively, the concentration of ion species $i$ as $\phi_{\mathrm{B}}=0$ and the electrical potential at surface $(r$ $=0$ or $r=H)$. Since both surfaces are at a constant charge density, the boundary conditions associated with Eqs. [9][11] are

$$
\begin{aligned}
& \xi_{\mathrm{con}}=\exp \left(-a \psi_{0}\right) \text { and } \xi_{\mathrm{co}}=\exp \left(b \psi_{0}\right), \eta=0, \\
& \frac{d \psi}{d \eta}=\frac{-\sigma_{0} \kappa \tau^{1 / 2}}{\rho_{0}(a+b)}, \eta=0 \\
& \xi_{\mathrm{con}}=\exp \left(-a \psi_{\mathrm{H}}\right) \text { and } \xi_{\mathrm{co}}=\exp \left(b \psi_{\mathrm{H}}\right), \eta=\frac{H \kappa}{\tau^{1 / 2}} \\
& \frac{d \psi}{d \eta}=\frac{\sigma_{H} \kappa \nu^{1 / 2}}{\rho_{0}(a+b)}, \eta=\frac{H \kappa}{\tau^{1 / 2}}
\end{aligned}
$$

where Eqs. [13b] and [13d] imply that the surface charge densities remain constant. In these expressions, $\sigma_{0}$ and $\sigma_{H}$ denote, respectively, the surface charge density at $r=0$ and at $r=H, \psi_{0}$ and $\psi_{H}$ the scaled electrical potential at $r=0$ and at $r=H$, respectively.

The numerical scheme for the resolution of Eqs. [9][11] is given in Appendix A.

\section{RESULTS AND DISCUSSION}

The dynamic behavior of the system under consideration is examined through numerical simulation. The system is at equilibrium initially. A change in the surface conditions due to, for example, the adsorption of potential determining ions onto the surfaces, is assumed, and the temporal distributions of electrical potential and ions are calculated. The result of numerical calculation reveals that for two same surfaces under the conditions $\sigma_{0}=\sigma_{H}=1.675 \times 10^{-3} \mathrm{coul} / \mathrm{m}^{2}, D_{\text {con }}$ $=D_{\text {co }}=10^{-9} \mathrm{~m}^{2} / \mathrm{s}, I=10^{-3} M, \epsilon_{\mathrm{r}}=78, T=298.15 \mathrm{~K}$, $H=24 \mathrm{~nm}$, and 1:1 electrolyte, the potential and space charge distributions approach a new equilibrium state at about $64 \times 10^{-9} \mathrm{~s}(\tau=0.692)$ for the case of $20 \%$ reduction in the surface charge density. This time scale is found to be insensitive to the valence of electrolyte.

The variation of the time to reach a new equilibrium distribution for ions, $t_{\mathrm{eq}}$, as a function of separation distance be-

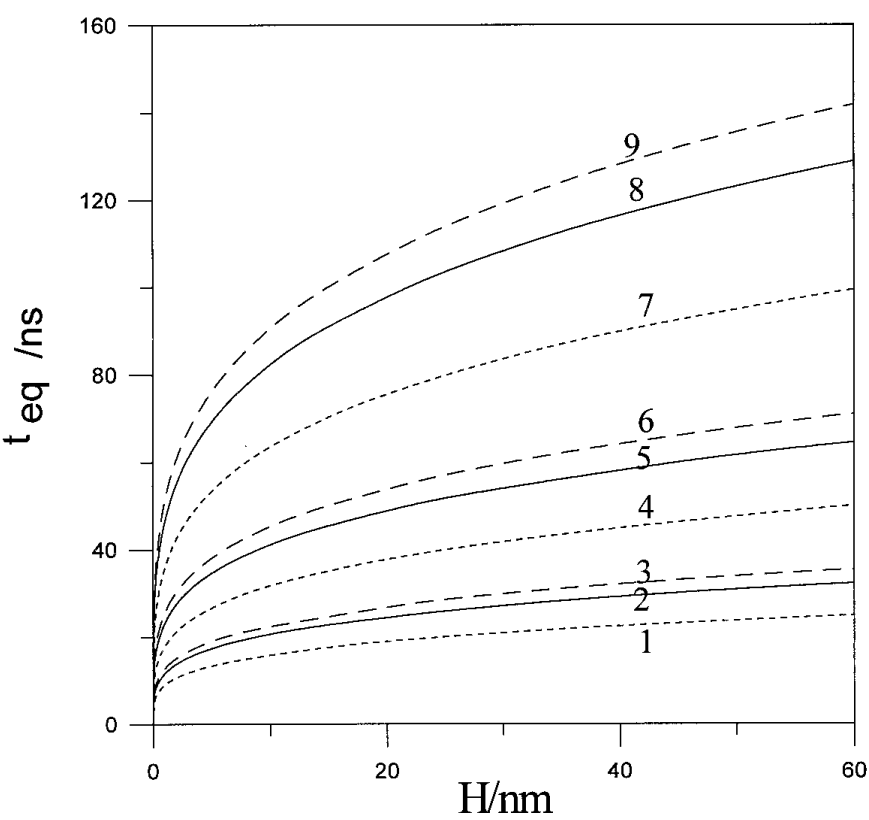

FIG. 1. Variation of the time to reach equilibrium distribution for ions, $t_{\text {eq }}$, as a function of separation distance between two surfaces, $H . t_{\text {eq }}$ is defined as the time at which $100 \%\left[\psi(r=H / 2)-\psi_{\mathrm{e}}(r=H / 2)\right] / \psi_{\mathrm{e}}(r=$ $H / 2)<1 \%, \psi_{\mathrm{e}}$ being the scaled equilibrium potential, and $\sigma_{0}$ is reduced by $20 \%$. Curve $1: D=2 \times 10^{-9} \mathrm{~m}^{2} / \mathrm{s}, I=2 \times 10^{-3} M, \sigma_{0}=1.675 \times$ $10^{-3} \mathrm{C} / \mathrm{m}^{2}$. Curve $2: D=2 \times 10^{-9} \mathrm{~m}^{2} / \mathrm{s}, I=10^{-3} M, \sigma_{0}=1.675 \times 10^{-3}$ $\mathrm{C} / \mathrm{m}^{2}$. Curve 3: $D=2 \times 10^{-9} \mathrm{~m}^{2} / \mathrm{s}, I=10^{-3} M, \sigma_{0}=3.35 \times 10^{-2} \mathrm{C} /$ $\mathrm{m}^{2}$. Curve 4: $D=10^{-9} \mathrm{~m}^{2} / \mathrm{s}, I=2 \times 10^{-3} M, \sigma_{0}=1.675 \times 10^{-3} \mathrm{C} / \mathrm{m}^{2}$. Curve 5: $D=10^{-9} \mathrm{~m}^{2} / \mathrm{s}, I=10^{-3} M, \sigma_{0}=1.675 \times 10^{-3} \mathrm{C} / \mathrm{m}^{2}$. Curve 6: $D=10^{-9} \mathrm{~m}^{2} / \mathrm{s}, I=10^{-3} M, \sigma_{0}=3.35 \times 10^{-2} \mathrm{C} / \mathrm{m}^{2}$. Curve $7: D=$ $5 \times 10^{-10} \mathrm{~m}^{2} / \mathrm{s}, I=2 \times 10^{-3} M, \sigma_{0}=1.675 \times 10^{-3} \mathrm{C} / \mathrm{m}^{2}$. Curve $8: D$ $=5 \times 10^{-10} \mathrm{~m}^{2} / \mathrm{s}, I=10^{-3} M, \sigma_{0}=1.675 \times 10^{-3} \mathrm{C} / \mathrm{m}^{2}$. Curve 9: $D=$ $5 \times 10^{-10} \mathrm{~m}^{2} / \mathrm{s}, I=10^{-3} M, \sigma_{0}=3.35 \times 10^{-2} \mathrm{C} / \mathrm{m}^{2}$.

tween two particles for various values of the parameters of the system under consideration is presented in Fig. 1. Here, $t_{\mathrm{eq}}$ is defined as the time at which the deviation of the concentration of ions at the midpoint between two particles from the corresponding new equilibrium value is smaller than $1 \%$. For the present system, the midpoint is the position that has the farthest distance from surface, and receives the least influence from both surfaces. If the system reaches equilibrium at this point, it reaches equilibrium at other points. Also, the midpoint is not only a natural choice for the reference point but also plays a significant rule in the evaluation of the electrical interactions between two planar surfaces $(13-16)$. It can be shown that it takes infinite time for ions to reach new equilibrium distributions. Here, the criterion of $1 \%$ deviation from a new equilibrium state is chosen arbitrary. Figure 1 shows that the following conditions will lead to a longer $t_{\mathrm{eq}}$ : (a) small ionic strength (thick double layer), (b) small mean diffusivity, (c) large separation distance, and (d) large surface charge density. The first and the second of these two are expected, since the relaxation time for ions is inversely proportional to its diffusivity, and is proportional to $\left(1 / \kappa^{2}\right)$. In the third condition, since ions 
need to diffuse through a longer distance, it is expected that $t_{\text {eq }}$ is longer. Finally, an increase in the surface charge density results in an increase in the electric field, and therefore, an increase in the driving force for ions to reach the new equilibrium distribution. On the other hand, a greater surface charge density implies that a greater amount of ions need to migrate. The net effect is that $t_{\mathrm{eq}}$ increases with $\sigma_{0}$. This effect, however, is not pronounced: a twentyfold increase in $\sigma_{0}$ leads to about $10 \%$ increase in $t_{\text {eq }}$.

The question arises as what is the relative significance of the time scale for double-layer relaxation compared to that for a Brownian encounter between two interacting particles. If the latter is much greater than the former, since the rate of response of the ions in a double layer is much faster than that of the approaching of particles, an equilibrium double layer can be assumed. If $H \kappa>2$, the Brownian motion is due to the diffusion of particle over a distance $2 / \kappa$; if $H \kappa$ $<2$, the motion is slowed down by hydrodynamic retardation. The time scale for Brownian encounters, $t_{\mathrm{Br}}$, can be estimated by $(8,17)$

$$
t_{\mathrm{Br}} \cong\left\{\begin{array}{l}
\frac{12 \pi \mu a_{\mathrm{c}}}{\kappa^{2} k_{\mathrm{B}} T W}, H \kappa>2, \\
\frac{3 H^{2} \pi \mu a_{\mathrm{c}}}{k_{\mathrm{B}} T W}, H \kappa<2,
\end{array}\right.
$$

where $W=\left(1+2 a_{\mathrm{c}} / 3 H\right) /\left[1+\left(13 a_{\mathrm{c}} / 6 H\right)+\left(a_{\mathrm{c}}^{2} / 3 H^{2}\right)\right]$, $a_{\mathrm{c}}$ being the radius of a particle, $\mu$ is the viscosity of fluid, and $k_{\mathrm{B}}$ is the Boltzmann constant. The time scale for doublelayer relaxation by ionic diffusion, $t_{\mathrm{dl}}$, can be expressed by

$$
t_{\mathrm{dl}} \cong\left\{\begin{array}{l}
\frac{(2 / \kappa)^{2}}{D}, H \kappa>2 \\
\frac{H^{2}}{D}, H \kappa<2
\end{array}\right.
$$

Therefore a measure for the significance of the dynamic behavior of double-layer relaxation is $t_{\mathrm{dl}}>t_{\mathrm{Br}}$. Equations [14] and [15] lead to

$$
D a_{\mathrm{c}}<\left(\frac{\kappa_{\mathrm{B}} T W}{3 \pi \mu}\right)
$$

Figure 2 shows the simulated result based on this expression. Region (1) denotes the case the double-layer relaxation is more significant than the Brownian motion; in region (2) the reverse is true. The boundary between these regions denotes the case where the time scales for double-layer relaxation and Brownian motion are comparable. Note that if $\mathrm{H} /$ $a_{\mathrm{c}} \rightarrow \infty$, we have isolated particles and free ion diffusion. On the other hand, if $H / a_{\mathrm{c}} \rightarrow 0$, Eq. [16] leads to $D \rightarrow 0$.

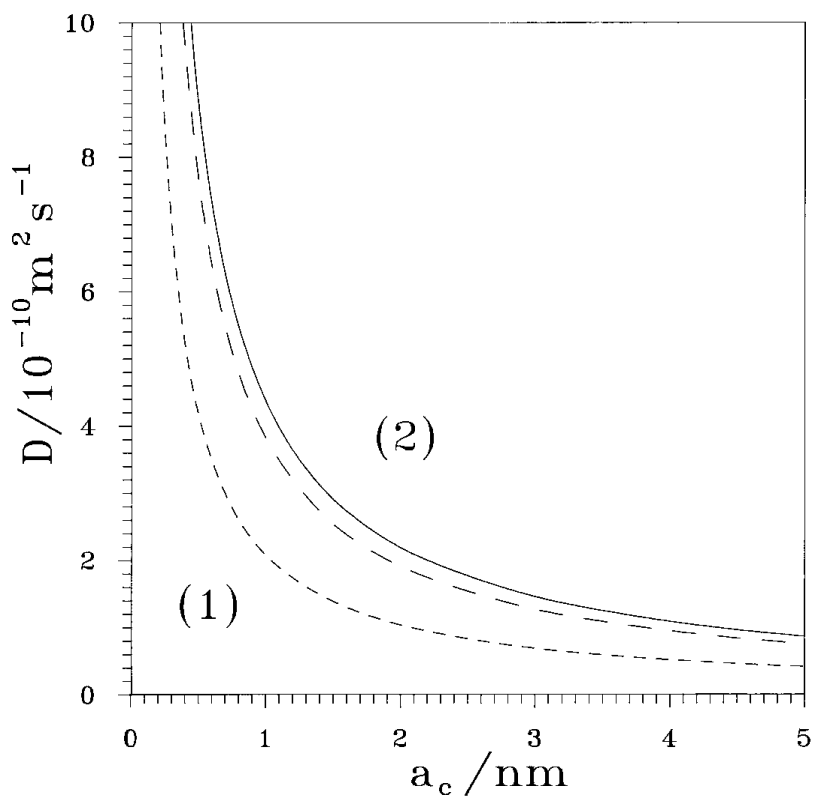

FIG. 2. Simulated results which show the relative significance of the time scales for double-layer relaxation and Brownian encounter. Region (1) denotes the case the former is more significant than the latter; the reverse is true in region (2). The boundary between regions (1) and (2), represented by solid or dashed lines, is described by Eq. [16] with equal sign. (-) $H / a_{\mathrm{c}} \rightarrow \infty ;(--) H / a_{\mathrm{c}}=10 ;(--) H / a_{\mathrm{c}}=1 ; T=298.15 \mathrm{~K}$, and $\mu=10^{-3} \mathrm{~Pa} \mathrm{~s}$.

This means that the diffusion of ions is infinitely slow. As can be seen from Fig. 2, the higher the ratio $H / a_{\mathrm{c}}$, the greater the region in which the kinetic behavior of double-layer relaxation is significant.

Due to the interaction between two particles, there will be a relative motion between them. It can be shown that the scaled separation distance between two particles, $H_{\mathrm{c}}$, is described by (Appendix C)

$$
\frac{\partial^{2} H_{\mathrm{c}}}{\partial t^{2}}+\frac{9 \mu \beta}{a_{\mathrm{c}}^{2} \rho} \frac{d H_{\mathrm{c}}}{d t}=\frac{3 R T}{2 \pi a_{\mathrm{c}}^{5} \rho} \frac{\partial \omega_{\mathrm{D}}}{\partial H_{\mathrm{c}}},
$$

where $\rho$ is the density of a particle and $\beta, H_{\mathrm{c}}$, and $\omega_{\mathrm{D}}$ are defined in Eqs. [C2a], [C2c], and [C6], respectively.

Figure 3 illustrates the simulated temporal variation in the separation distance between two particles; the corresponding variation in the electrostatic interaction force between particles is presented in Fig. 4. Also shown in these figures are the results for the corresponding equilibrium model. Both constant surface potential model and constant surface charge density model are examined. A brief summary for the numerical procedure adopted is given in Appendix B. Figure 3 reveals that the rate of approach of two particles is faster if the surfaces are maintained at constant potential than if the surfaces are maintained at constant charge density. The dynamic behavior of the relaxation of ions in the double layers 
is found to have the effect of retarding the motion of particle. This is consistent with the results of Lyklema (9) and Dukhin and Lyklema (10). Figure 4 shows that the electrostatic repulsion force for constant surface charge density model is greater than that for constant surface potential model. This is because the latter allows the regulation of charges near the surfaces. The electrostatic repulsion force for a dynamic model is greater than that for the corresponding equilibrium model. This is expected since the concentration of counterions near the surface of the latter is greater than that of the former. When the relative position of two interacting particles varies, the distributions of ions in the double layers vary accordingly. If the movement of particles is sufficiently fast, the deviations of the distributions of ions from the corresponding equilibrium values can be appreciable. According to Fig. 3, for constant potential models, when the equilibrium model predicts that two particles encounter each other, the dynamic model suggests that the distance between two particles is about $19 \%$ of the original distance. For constant surface charge models, when the equilibrium model predicts that two particles encounter each other, the dynamic model suggests that the distance between two particles is about $28 \%$ of the original distance. The deviation in the contact time of the equilibrium model from the corresponding dynamic model is about $8 \%$ for constant potential models, and about $11 \%$ for constant surface charge models. The deviation

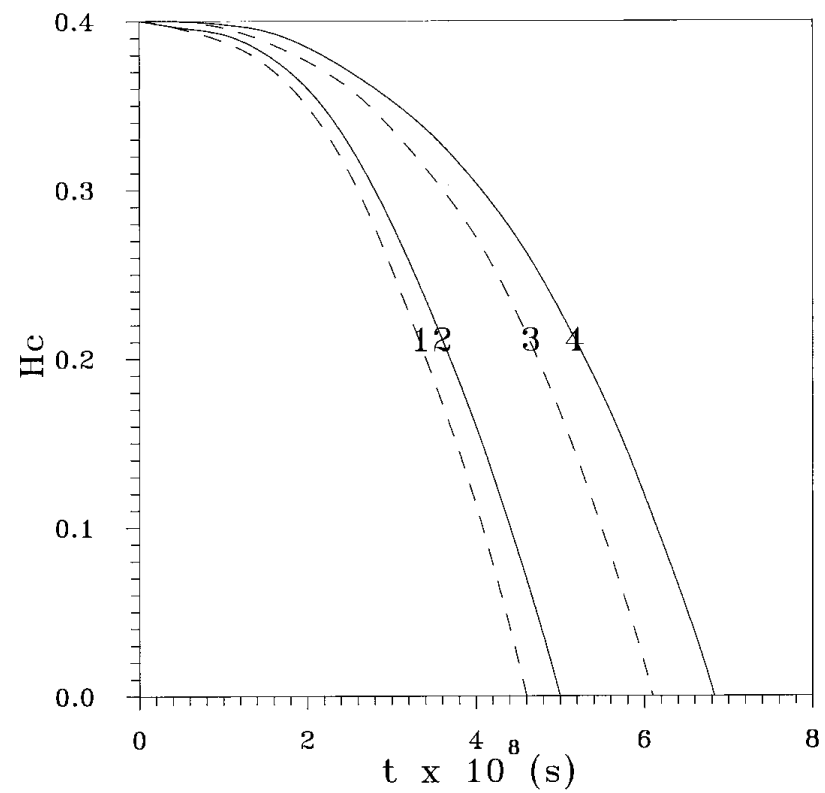

FIG. 3. Temporal variation in the separation distance between particles for the case $I=0.1 M, a=2, b=1, a_{\mathrm{c}}=2 \times 10^{-9} \mathrm{~m}, A=1 \times 10^{-19}$ $\mathrm{J}, \rho=10^{3} \mathrm{~kg} / \mathrm{m}^{3}, \epsilon_{\mathrm{r}}=78, T=298.15 \mathrm{~K}$, and $\mu=10^{-3} \mathrm{~Pa} \mathrm{~s}$. The initial condition is $H_{\mathrm{c}}=0.4$ and $v=0$. Solid curves, dynamic model with $D_{\text {con }}$ $=D_{\text {co }}=10^{-9} \mathrm{~m}^{2} / \mathrm{s}$; dashed curves, equilibrium model. Curves 1 and 2 , constant surface potential with $\phi(t=0)=25.5 \mathrm{mV}$; curves 3 and 4 , constant surface charge density with $\phi^{\prime}(t=0)$ corresponds to $\phi(t=0)$ $=25.5 \mathrm{mV}$. The system is initially at equilibrium.

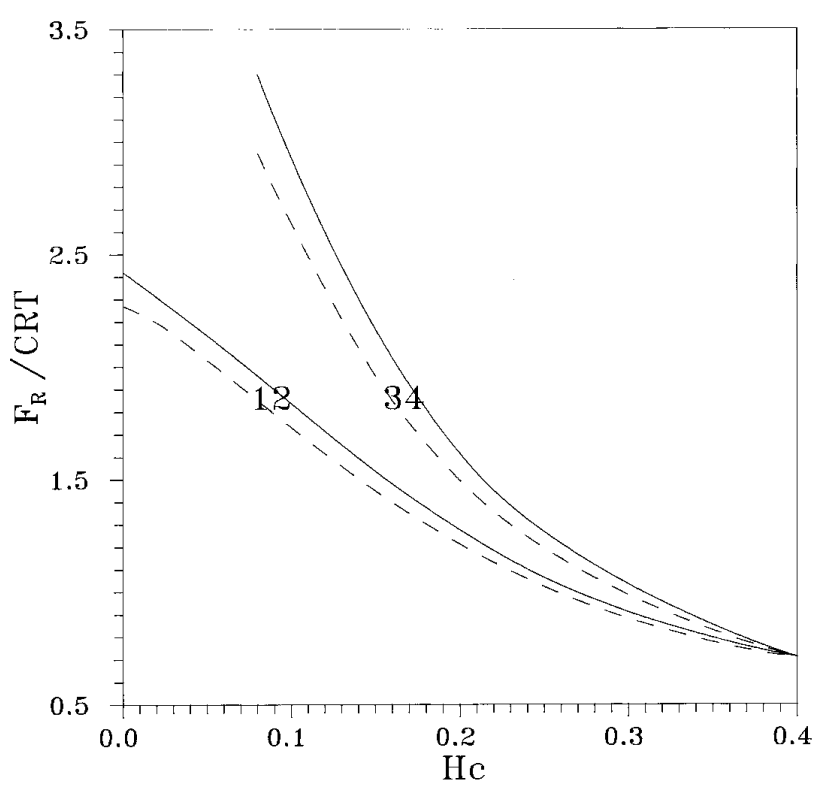

FIG . 4. Variation of interaction force as a function of mean separation distance between particles for the case of Fig. 3 .

in the contact time between two particles is a cumulated effect of the dynamic behavior of the interacting double layers.

\section{APPENDIX A}

Since the system is symmetric about $r=H / 2$, only the distributions of potential and ion concentrations in the range $0<r<H / 2$ need to be solved. Suppose that the values of $\xi_{\text {con }}$ and $\xi_{\text {co }}$ at time $t$ are given. The following procedure is employed.

Step 1. Substitute $\xi_{\text {con }}$ and $\xi_{\text {co }}$ into Eq. [11] and solving the resultant expression numerically subject to Eq. [13b] and an assumed $\psi_{0}$. If the electrical potential thus obtained is symmetric about $r=H / 2$, the assumed $\psi_{0}$ is correct. Otherwise, a new $\psi_{0}$ is assumed. This step is repeated until the symmetric condition for electrical potential is satisfied.

Step 2. Substitute the electrical potential obtained in step 1 into Eqs. [9] and [10] and advance time to $t+\Delta t, \Delta t$ being an increment in the time scale.

Step 3. Evaluate $\xi_{\text {con }}(r=0)$ and $\xi_{\text {co }}(r=0)$ through Eq. [13a].

Step 4. Assume the values of the derivatives of $\xi_{\text {con }}$ and $\xi_{\text {co }}$ at $r=0, \xi_{\text {con }}^{\prime}(r=0)$ and $\xi_{\text {co }}^{\prime}(r=0)$, such that the solutions to Eqs. [9] and [10] satisfy the symmetric condition $\xi_{\text {con }}^{\prime}(r=\mathrm{H} / 2)=0$ and $\xi_{\text {co }}^{\prime}(r=\mathrm{H} / 2)=0$. When this is completed, go back to step 1 .

This procedure is continued until the solutions to Eqs. [9]-[11] converge. This yields $\xi_{\text {con }}, \xi_{\text {co }}$, and $\psi$ at time $t+\Delta t$. 


\section{APPE NDIX B}

The temporal variations in the distance and the electrical interaction force between two particles at constant charge density are calculated through the numerical procedure below.

Step 1. At a specific time $t_{\mathrm{k}}$ and position $H_{\mathrm{c}, \mathrm{k}}$, Eqs. [9][11] are solved numerically subject to the boundary conditions described by Eqs. [13a]-[13d] and a set of guessed scaled surface potential $\psi_{0, \mathrm{~g}}$ and scaled differential ion concentrations, $\xi_{\mathrm{con}, 0, \mathrm{~g}}^{\prime}$ and $\xi_{\mathrm{co}, 0, \mathrm{~g}}^{\prime}$, for the particle at the origin.

Step 2. The position at which the estimated scaled potential $\psi_{\mathrm{c}}\left(H_{\mathrm{c}, \mathrm{k}}\right)$ and scaled differential ion concentrations $\xi_{\mathrm{con}, 0, \mathrm{~g}}^{\prime}\left(H_{\mathrm{c}, \mathrm{k}}\right)$ and $\xi_{\mathrm{co}, 0, \mathrm{~g}}^{\prime}\left(H_{\mathrm{c}, \mathrm{k}}\right)$ are close enough to $\psi_{0, \mathrm{~g}}$, $\xi_{\mathrm{con}, 0, \mathrm{~g}}^{\prime}$, and $\xi_{\mathrm{co}, 0, \mathrm{~g}}^{\prime}$ is defined as $x_{1}$. If such an $x_{1}$ does not exist, return to step 2 with newly guessed $\psi_{0, \mathrm{~g}}, \xi_{\mathrm{con}, 0, \mathrm{~g}}^{\prime}$, and $\xi_{\mathrm{co}, 0, \mathrm{~g}}^{\prime}$.

Step 3. The scaled electrical interaction force between two particles is calculated by Eq. [C3], and the result is substituted into Eq. [C4] to evaluate $\omega_{\mathrm{el}}$. Add $\omega_{\mathrm{el}}$ to $\omega_{\mathrm{vdw}}$ for particles separated by $x_{1}$ to obtain $\omega_{\mathrm{D}}$.

Step 4. Solving Eq. [17] subject to the initial conditions $H=H_{\mathrm{c}, \mathrm{k}-1}$ and $v=v_{\mathrm{k}-1}$ at $t=t_{\mathrm{k}-1}$ we obtain the distance $H=x_{2}$ at $t=t_{\mathrm{k}}$. Here, we assume that $H_{0}=2 a_{\mathrm{c}}$ and $v_{0}=$ 0 at $t_{0}=0, v_{i}$ is the velocity of particle. If $x_{1}$ and $x_{2}$ are inconsistent, return to step 1 with newly guessed $\psi_{0, \mathrm{~g}}$, $\xi_{\mathrm{con}, 0, \mathrm{~g}}^{\prime}$, and $\xi_{\mathrm{co}, 0, \mathrm{~g}}^{\prime}$. The procedure is repeated until the difference between $x_{1}$ and $x_{2}$ is smaller than a prespecified value.

Step 5. The time is advanced by $t_{\mathrm{k}+1}=t_{\mathrm{k}}+\Delta t, \Delta t$ being a prespecified time interval, and the procedure is repeated by returning to step 1 .

The numerical procedure for the case of constant surface potential is similar to that described above except that Eqs. [13b] and [13d] are replaced by $\psi=\psi_{0}$ at both $\eta=0$ and $\eta=H \kappa / \tau^{1 / 2}$. Note that the scaled differential potentials on both surfaces are of the same magnitude but are opposite in sign.

\section{APPENDIX C}

Suppose that the total interaction force between two particles is composed of the DLVO force $F_{\mathrm{D}}$ and the hydrodynamic force $F_{\mathrm{H}}$. The former includes the electrical force and the van der Waals force. We have

$$
m\left(\frac{d v}{d t}\right)=F_{\mathrm{D}}+F_{\mathrm{H}}
$$

where $m$ and $v$ denote, respectively, the mass and the velocity of a particle. Since only one-dimensional motion of the particles is considered, all the vectors are replaced by the corresponding scalars. Here we assume that the expected net ef- fect due to any random force vanishes. For convenience, we let the coordinates move with one of the two particles.

The hydrodynamic force between two spherical particles can be expressed as (18)

$$
F_{\mathrm{H}}=-6 \pi \mu a_{\mathrm{c}} \beta v,
$$

where

$$
\begin{aligned}
& \beta=\frac{4}{3}[\sinh (\alpha)] \sum_{n=0}^{\infty}\left\{\frac{n(n+1)}{(2 n-1)(2 n+3)}\right. \\
&\left.\times\left[\frac{4 \cosh ^{2}\left[\left(n+\frac{1}{2}\right) \alpha\right]+(2 n+1)^{2} \sinh ^{2}(\alpha)}{2 \sinh [(2 n+1) \alpha]-(2 n+1) \sinh (2 \alpha)}-1\right]\right\}, \\
& \alpha=\cosh ^{-1}\left(1+H_{\mathrm{c}}\right), \\
& H_{\mathrm{c}}=H / a_{\mathrm{c}} .
\end{aligned}
$$

Here we assume that an electrical double layer is thin compared with the radius of a particle, and, therefore, the former can be treated as a planar surface.

For the present dynamic system, the electrical force per unit area between two particles $F_{\mathrm{R}}$ can be calculated by (6)

$$
\frac{F_{\mathrm{R}}}{C R T}=-\left[\frac{a+b}{2 \chi^{2}}\left(\frac{\partial \psi}{\partial X}\right)^{2}+\int_{0}^{\psi}\left(\xi_{\mathrm{con}}-\xi_{\mathrm{co}}\right) d \psi\right] \text {, }
$$

where $\psi$ is the scaled electrical potential, $\chi=\kappa a_{\mathrm{c}}, X=r /$ $a_{\mathrm{c}}$, and $C=a C_{\mathrm{a}}^{0}=b C_{\mathrm{b}}^{0}$. The scaled electrical potential energy between two particles $\omega_{\mathrm{el}}$ can be estimated by (6)

$$
\omega_{\mathrm{el}}=2 \pi a_{\mathrm{c}}^{3} \int_{H_{\mathrm{c}}}^{\infty} \int_{\ell}^{\infty} \frac{F_{\mathrm{R}}(\lambda)}{R T} d \lambda d \ell,
$$

where $\lambda$ and $\ell$ are dummy variables. The scaled van der Waals potential energy $\omega_{\mathrm{vdw}}$ can be evaluated by (19)

$$
\begin{aligned}
\omega_{\mathrm{vdw}}= & \frac{-A}{3 R T}\left\{\frac{1}{H_{\mathrm{c}}\left(H_{\mathrm{c}}+4\right)}+\frac{1}{\left(H_{\mathrm{c}}+2\right)^{2}}\right. \\
& \left.+\frac{1}{2} \ln \left[\frac{H_{\mathrm{c}}\left(H_{\mathrm{c}}+4\right)}{\left(H_{\mathrm{c}}+2\right)^{2}}\right]\right\},
\end{aligned}
$$

where $A$ is the Hamaker constant for the present particleelectrolyte solution-particle system. The scaled DLVO potential energy $\omega_{\mathrm{D}}$ is

$$
\omega_{\mathrm{D}}=\omega_{\mathrm{el}}+\omega_{\mathrm{vdw}}
$$

The corresponding DLVO force can be calculated by 


$$
F_{\mathrm{D}}=-\frac{R T}{a_{\mathrm{c}}}\left(\frac{\partial \omega_{\mathrm{D}}}{\partial H_{\mathrm{c}}}\right)
$$

\section{ACKNOWLEDGMENT}

This work is supported by the National Science Council of the Republic of China under Grant NSC83-0402-E002-001.

\section{REFERENCES}

1. Ninham, B. W., and Parsegian, V. A., J. Theor. Biol. 31, 405 (1971).

2. Chang, Y. I., and Hsu, J. P., J. Theor. Biol. 147, 509 (1990).

3. van Aken, G. A., Lekkerkerker, H. N. W., Overbeek, J. Th. G., de Bruyn, P. L., J. Phys. Chem. 94, 8468 (1990).

4. Hsu, J. P., and Kuo, Y. C., J. Chem. Soc., Faraday Trans. 89, 1229 (1993).

5. Kijlstra, J., and van Leeuwen, H. P., J. Colloid Interface Sci. 160, 424 (1993).
6. Hsu, J. P., Kuo, Y. C., and Chang, Y. I., Colloid Polym. Sci. 272, 946 (1994).

7. Overbeek, J. Th. G., J. Colloid Interface Sci. 58, 408 (1977).

8. Lyklema, J., Pure Appl. Chem. 52, 1221 (1980).

9. Lyklema, J., in "The Structure, Dynamics and Properties of Colloidal Systems"' (D. M. Bloor and E. Wyn-Jones, Eds.). Kluwer, The Netherlands, 1990.

10. Dukhin, S. S., and Lyklema, J., Langmuir 3, 94 (1987).

11. Shulepov, S. Yu., Dukhin, S. S., and Lyklema, J., J. Colloid Interface Sci. 171, 340 (1995).

12. Bluman, G. W., and Cole, J. D., "Similarity Methods for Differential Equations.', Springer-Verlag, New York, 1974.

13. Chan, D. Y. C., Pashley, R. M., and White, L. R., J. Colloid Interface Sci. 77, 283 (1980).

14. Kuo, Y. C., and Hsu, J. P., J. Colloid Interface Sci. 156, 250 (1993).

15. Hsu, J. P., and Kuo, Y. C., J. Colloid Interface Sci. 171, 483 (1995).

16. Hsu, J. P., and Kuo, Y. C., Colloid Polym. Sci. 273, 881 (1995).

17. Honig, E. P., Roebersen, G. J., and Wiersema, P. H., J. Colloid Interface Sci. 36, 97 (1971).

18. Happel, J., and Brenner, H., "Low Reynolds Number Hydrodynamics." Martinus Nijhoff, Boston, 1983.

19. Hunter, R. J., “Foundations of Colloid Science.' Oxford Univ. Press, Oxford, 1989. 